\title{
Avaliação e manejo da dor na criança: percepção da equipe de enfermagem
}

\author{
Assessment and management of pain in child: perception of the \\ nursing team
}

\author{
Débora Guedelha Blasii ${ }^{1}$; Ligyana Korki de Candido²; Mauren Teresa Grubisich \\ Mendes Tacla ${ }^{3}$; Rosângela Aparecida Pimenta Ferrari ${ }^{4}$
}

\begin{abstract}
Resumo
Esta pesquisa objetivou analisar a percepção da equipe de enfermagem quanto à avaliação e manejo da dor realizados em um setor de internação pediátrica. Trata-se de uma pesquisa qualitativa, observacional, prospectiva e transversal. Os dados foram coletados por meio de entrevista semiestruturada com a equipe de enfermagem do setor de internação pediátrica de um hospital universitário da cidade de Londrina - Paraná, no período de junho a agosto de 2009, e analisados conforme Mayan. Participaram da pesquisa 31 profissionais, sendo 4 enfermeiros e 27 das demais categorias de enfermagem. Verificou-se que menos da metade dos profissionais participou de cursos de capacitação para avaliação da dor realizados frequentemente no hospital. Entretanto, a maioria afirma realizar a avaliação da dor como $5^{\circ}$ sinal vital em todas as crianças. A escala de faces é a mais utilizada pela equipe, embora apenas $44 \%$ tenha descrito corretamente a sua técnica de utilização. Outros profissionais ainda referiram não utilizar nenhuma escala para avaliar a dor. A principal dificuldade apontada pela equipe foi em relação à avaliação da dor em crianças menores. Quanto à avaliação da dor no setor de forma geral, a maioria acha que precisa melhorar, relatando que alguns profissionais não avaliam a dor em todos os horários, ou o fazem de forma incorreta. Ainda assim, todos consideraram a avaliação da dor de grande importância no cuidado da criança. Conclui-se que ainda falta conhecimento e conscientização sobre a dor na criança na equipe de enfermagem.
\end{abstract}

Palavras-chave: Enfermagem Pediátrica. Medição da dor. Criança. Percepção. Equipe de enfermagem.

\begin{abstract}
This study aimed to analyze the perception of the nursing team regarding the assessment and management of pain performed in a pediatric unit. This is a qualitative, observational, prospective, and cross-sectional study. Data were collected through semi-structured interviews with the nursing team from a pediatric unit of an university hospital in the city of Londrina - Paraná, in the period from June to August 2009, and analyzed according to Mayan. In total, 31 professionals of the nursing team participated in this study, 4 nurses and 27
\end{abstract}

\footnotetext{
${ }^{1}$ Enfermeira. Ex-Residente de Enfermagem em Saúde da Criança, Departamento de Enfermagem, Centro de Ciências da Saúde Universidade Estadual de Londrina, Paraná - Brasil.

${ }^{2}$ Enfermeira, graduada pela Universidade Estadual de Londrina. Graduação sanduíche na Vancouver Island University, Canadá. Residente de Enfermagem em Saúde da Criança, Departamento de Enfermagem, Centro de Ciências da Saúde - Universidade Estadual de Londrina, Paraná - Brasil.

${ }^{3}$ Enfermeira. Doutora em Enfermagem. Professor Adjunto e Vice-coordenadora da Residência em Enfermagem em Saúde da Criança do Departamento de Enfermagem, Centro de Ciências da Saúde, Universidade Estadual de Londrina, Paraná - Brasil.

${ }^{4}$ Enfermeira. Doutora em enfermagem. Professor adjunto e coordenadora da residência em enfermagem em Saúde da Criança, Departamento de Enfermagem, Centro de Ciências da Saúde, Universidade Estadual de Londrina, Paraná - Brasil.
} 
from other nursing categories. It was verified that less than half of the professionals attended training courses for pain assessment often carried out in this hospital. However, most stated to perform the assessment of pain as the 5 th vital sign for all children. The facial scale is the most used by the team, although only $44 \%$ had correctly described the technique of use. Other professionals also reported that they did not use any scale to assess pain. The main difficulty pointed by the nursing team was regarding assessment of pain in infants. Regarding the unit's assessment of pain in general, most of the professionals think that they need to improve, reporting that other professionals do not assess pain all the times, or do it incorrectly. Nevertheless, everyone considered pain assessment of major importance in childcare. It is concluded that there is still a lack of knowledge and awareness about child's pain in the nursing team.

Keywords: Pediatric Nursing. Pain measurement. Child. Perception. Nursing team.

\section{Introdução}

A dor é um dos sintomas mais comuns nas mais diversas enfermidades. Acompanha o homem desde o início dos tempos e é descrita pela Associação Internacional para o Estudo da Dor (IASP) como: "Uma experiência sensorial e emocional indesejada associada com lesão real ou potencial do tecido, ou descrita em termos de tal dano." (MERSKEY; BOGDUK, 2012, p. 211). A dor também é subjetiva, sendo "[...] aquilo que a pessoa que a sente diz que é, e existindo sempre que a pessoa assim o disser [...]." (MERSKEY; BOGDUK, 2012, p. 211). Entretanto, a impossibilidade do individuo expressar-se verbalmente não nega a possibilidade do mesmo estar experienciando a dor e necessitar de seu tratamento e alívio (MERSKEY; BOGDUK, 2012, p. 211-212).

O fenômeno da dor pode ser considerado o sintoma de alarme mais importante encontrado no corpo humano e está sempre relacionado a fatores sensoriais, afetivos, comportamentais, cognitivos e socioculturais, além da patologia de base. É, portanto, um fenômeno multifatorial (HOCKENBERRY, 2011).

Em relaçãoà dor na criança, só houve um aumento significativo dos estudos sobre esse tema a partir da década de 1980, quando alguns pesquisadores evidenciaram que as crianças são capazes de quantificar o seu sofrimento, desde que os adultos forneçam instrumentos adequados para isso. Apesar da busca pelo entendimento do mecanismo, acesso e manejo da dor infantil, pouco do conhecimento obtido em pesquisas tem sido observado na prática da enfermagem (TACLA; HAYASHIDA; LIMA, 2008; WALCO; GOLDSCHNEIDER, 2008).

O fato descrito anteriormente é preocupante, porque para as crianças, a experiência dolorosa torna-se ainda mais assustadora, primeiramente porque temores em relação a lesões corporais e dor prevalecem entre elas (HOCKENBERRY, 2011). Em segundo lugar, pela dificuldade que alguns profissionais de saúde têm de identificar, mensurar e controlar a dor na criança, uma vez que, dependendo do nível cognitivo em que esta se encontra, não consegue expressar-se verbalmente, e cabe à equipe de saúde avaliar a dor apenas por suas reações e comportamentos. Dessa maneira, é fundamental que os profissionais utilizem instrumentos adequados para avaliação da dor, compatíveis com a idade e o desenvolvimento cognitivo da criança, além de condizentes com sua condição clínica e neurológica (KANAI; FIDELIS, 2010; LEÃO et al., 2008; PERSEGONA; ZAGONEL, 2008).

No caso da criança hospitalizada, o fenômeno da dor toma proporções ainda maiores. A combinação "dor e hospitalização" pode resultar em um nível maior de ansiedade e medo, ou até mesmo a quadros depressivos, conforme a situação. A equipe de enfermagem, nesses casos, deve estar alerta e preparada para dar todo o suporte necessário; e para que isso aconteça, deve intervir no reconhecimento, avaliação e tratamento da dor, buscando o seu alívio e melhorando a qualidade de vida e adaptação da criança, de maneira ética e humanizada (KANAI; 
FIDELIS， 2010; PERSEGONA； ZAGONEL， 2008).

Estudos demonstram que a equipe de enfermagem tem a percepção de que a criança hospitalizada vivencia situações potencialmente dolorosas, como procedimentos invasivos, a separação da criança dos pais, dor crônica relacionada à própria doença da criança (como anemia falciforme, pneumonia, fraturas e neoplasias) (CARDIM et al., 2009; PAIXÃO et al., 2011; SILVA et al., 2010). Mesmo assim, crenças errôneas a respeito do assunto são constantes, o que compromete diretamente a assistência na avaliação e manejo dessa dor. Entre os mitos comuns encontramos: a criança sente menos dor que o adulto, a criança se acostuma ou tolera a dor mais facilmente que o adulto, o uso de opióides pode ser viciante, a avaliação da dor na criança é difícil e despende muito tempo, entre outras (TACLA; HAYASHIDA; LIMA, 2008; FINLEY; 2010).

$\mathrm{Na}$ verdade, a criança sente dor tanto quanto um adulto ou até mais, visto que possui vias da dor e sistemas neuroquímicos associados à transmissão e modulação da dor intactos e funcionantes, por outro lado, seus mecanismos de controle inibitório da dor ainda estão imaturos (LEÃO et al., 2008; WHALEY; WONG, 2007). Ao contrário do que se pensa, a criança possui memória para a dor e poderá evitar cuidados médicos futuros, caso tenha vivenciado situações dolorosas não tratadas em sua infância (FINLEY et al., 2005).

Quanto à avaliação da dor, deve-se lembrar que a criança não é um adulto em miniatura, portanto parâmetros de adultos não podem ser usados para clientes pediátricos. Talvez por conta disso as crianças recebam intervenções de controle da dor com menos sucesso do que os adultos. Dessa maneira, a utilização de instrumentos adequados para a avaliação da dor é o primeiro passo para um tratamento efetivo, seja ele farmacológico, não farmacológico ou a associação desses (KAZANOWSKY; LACCETTI, 2005). Atualmente, os instrumentos de avaliação da dor infantil podem ser uni ou multidimensionais (LEÃO et al., 2008), e divididos em três categorias: autorrelato, observação do comportamento e instrumentos compostos de medida de dor (FINLEY, 2010).

$\mathrm{Na}$ instituição onde foi realizada esta pesquisa, a dor foi implantada como $5^{\circ}$ sinal vital no ano de 2007. Para tanto foram realizados cursos de capacitação, abordando separadamente a temática "dor na criança" e "dor no adulto" para toda a equipe de saúde (NASCIMENTO; KRELING, 2011). Para o uso no setor de pediatria, foram preconizadas pela instituição a Escala de Faces de Claro (CLARO, 1993), e a Neonatal Infant Pain Score (NIPS) (LAWRENCE et al., 1993). Porém, o que se tem observado é que a avaliação da dor na criança, na maioria das vezes, não é realizada ou o é realizada de forma incorreta.

Diante deste panorama, esta pesquisa teve como objetivo analisar a percepção da equipe de enfermagem quanto à avaliação e ao manejo da dor realizado em um setor de internação pediátrica de um hospital universitário.

\section{Material e Método}

Trata-se de um estudo qualitativo, observacional, prospectivo, de ordem transversal. A coleta de dados foi realizada em um setor de internação pediátrico de um hospital universitário da cidade de Londrina - Paraná, durante o período de junho a agosto de 2009. O setor em questão possui 32 leitos pediátricos, sendo 10 leitos para pacientes cirúrgicos e 22 leitos para pacientes clínicos, atendendo crianças de 0 (zero) a 12 anos com as mais diversas patologias. A equipe de enfermagem é composta por 37 funcionários sendo 5 enfermeiros, 22 auxiliares e 6 técnicos de enfermagem e 4 auxiliares operacionais. O critério de inclusão foi: ser membro da equipe de enfermagem da referida unidade. E os critérios de exclusão foram: estar de licença ou férias e recusa na participação. Ao final, 
31 profissionais aceitaram participar do estudo, sendo 4 enfermeiros e 27 das demais categorias. Dos seis funcionários não incluídos na pesquisa, três estavam de licença médica prolongada e três recusaram-se a participar.

Os dados foram coletados com a utilização de uma entrevista semiestruturada, com a questão norteadora: "você realiza a avaliação da dor como $5^{\circ}$ sinal vital?". Também foram empregadas questões auxiliares, como: importância da avaliação da dor na criança hospitalizada; dificuldades ao avaliar a dor; opinião sobre a avaliação da dor realizada pela equipe de enfermagem no setor; uso de medidas não farmacológicas para alívio dor. Além de questões sociodemográficas.

Os funcionários foram entrevistados após serem informados sobre os objetivos da presente pesquisa e assinarem o Termo de Consentimento Livre e Esclarecido, cientes de que poderiam desistir da participação a qualquer momento e de que seus dados pessoais seriam mantidos no mais absoluto sigilo. As falas dos sujeitos foram gravadas e, após transcrição e análise, as gravações foram apagadas; da mesma forma, os nomes dos participantes foram substituídos por siglas (ENF, AUX, TEC e AOP) preservando assim a identidade dos entrevistados.

O método utilizado para análise de conteúdo dos dados obtidos nas entrevistas compreendeu as etapas de codificação, categorização e a integração dos dados em núcleos temáticos (MAYAN, 2001). A codificação teve início com a identificação das falas segundo a ordem de aparecimento na transcrição e o número do informante. Durante repetidas leituras do material, grifamos partes do texto, registramos nossas impressões, observações e questionamentos nas margens livres, ao lado dos parágrafos correspondentes. Segundo Mayan (2001), mediante a codificação o pesquisador apenas se familiariza com os dados e inicia a organização das informações.

Esta pesquisa foi devidamente avaliada e aprovada pelo Comitê de Ética em Pesquisa Envolvendo Seres Humanos da Universidade
Estadual de Londrina de acordo com o parecer $\mathrm{n}^{\mathrm{o}}$ 215/08, CAAE n ${ }^{\circ}$ 0211.0.268.000-08.

\section{Resultados e Discussão}

O tempo de serviço dos profissionais de enfermagem na unidade pediátrica variou de quatro meses a 33 anos, com uma média de 13 anos.

Após analise dos dados obtidos nas entrevistas, foram identificadas duas categorias: Avaliação da dor na criança: estratégias e dificuldades; Fragilidades no manejo da dor na criança.

\section{Avaliação da dor na criança: estratégias e} dificuldades:

Foi possível identificar que a escala mais aceita e utilizada foi a Escala de Faces de Claro (CLARO, 1993), mas ainda assim, apenas uma pequena parcela a utiliza corretamente.

\begin{abstract}
Ah, quando a criança é grande, eu mostro para ela as carinhas e explico cada carinha. A criança que não entende muito vai naquela parte que é feia, lá... Quando não entende eu falo: olha, esse aqui é bonito, bonito, bonito, e esse aqui é feio. Aí eles vão no bonitinho, porque não entende... o que quer dizer que a criança está bem. (Aux.1)
\end{abstract}

Esses dados chamam a atenção para a questão da falta de preparo, de atualização técnica e até mesmo de interesse dos profissionais de enfermagem, o que pode significaruma gravequebra daresponsabilidade ética de cada um (TACLA; HAYASHIDA; LIMA, 2008). A atualização da equipe deve ser continua, a fim de garantir uma assistência humanizada e de qualidade (SILVA et al., 2010). Antes de confiar nos dados da avaliação, é necessário que a equipe se sinta segura e saiba como utilizar os instrumentos de avaliação. O instrumento só é válido quando medições são compatíveis com a realidade, e para que isso aconteça, os profissionais de saúde devem estar preparados para aplicá-lo (SILVA et al., 2007). 
Porém, mesmo demonstrando dificuldades durante a entrevista e descrevendo de forma errônea a técnica de avaliação da dor na criança, a maior parte da equipe referiu não ter problemas na hora de aplicar o método. Alguns acreditavam que o fato de possuírem experiência na área pediátrica ou de terem muitos filhos, dava-lhes mais experiência no assunto e, portanto tornava-os mais seguros para realizar a avaliação.

Dificuldade? Dificuldade... olha, eu não encontro dificuldade, eu não sei se é porque... olha deixa eu falar... sobre a dor, a vida toda eu mexi com criança sadia. Eu era professora de $1^{\mathrm{a}}$ a $4^{\mathrm{a}}$, e tenho 5 filhos... Então, eu não encontro dificuldade em avaliar a dor na criança. (AUX. 2)

Ah, dificuldade? Eu não sinto assim dificuldade para avaliar a dor de uma criança. Porque eu sou mãe, e eu acho assim, uma criança, quando ela não tem dor, ou ela está dormindo bem tranquila ou ela está sorrindo. Ela está sempre feliz. (AUX. 3)

Essa associação entre a avaliação da dor na criança hospitalizada com a experiência dolorosa dos próprios filhos do profissional de saúde também foi evidenciada em estudo realizado em uma unidade pediátrica de um hospital de natureza privada, da cidade de São Paulo, Brasil. Entretanto, essa relação se deu no sentido do maior envolvimento no cuidado da criança com dor. Ao trazer à tona as próprias vivências familiares, o profissional se sensibiliza e valoriza a dor da criança, como se fosse um membro de sua família (KANAI; FIDELIS, 2010). Por outro lado, a comparação também pode trazer o sentimento de impotência, e a assistência pode se tornar mais difícil quando a equipe de enfermagem não consegue lidar com seu sentimento de maneira ética e profissional. Portanto, esses sentimentos precisam ser transpostos de maneira que não interfiram no inter-relacionamento entre a equipe de enfermagem, a criança e a família (KAZANOWSKI; LACCETTI, 2005).

Quanto às dificuldades apontadas pela equipe, a maioria está relacionada à avaliação da dor em crianças menores, o que fica evidente em algumas falas.
É mais difícil quando é criança que não fala, né? Que são os menorzinhos... a gente nunca tem certeza absoluta do que eles tão sentindo. Quando fala é fácil de resolver o problema. (ENF1)

Olha, no geral não [não tem dificuldade]. Eu ainda fico meio... nos menorzinhos. Será que eu fui mesmo... avaliei de forma correta, eu fico meio insegura. Mas nos maiores eu acredito que não. (AUX4)

O auto-relato é a forma mais aceita e utilizada na hora de avaliar a dor de um paciente, porém é uma técnica que se baseia na habilidade do indivíduo de comunicar seus sintomas, talvez por isso haja uma maior dificuldade de avaliação quando se trata de crianças pequenas. As crianças pré-verbais, ou que não tem capacidade de comunicação verbal, não apresentam um auto-relato efetivo, e, portanto, a avaliação da dor pelos cuidadores nesses casos, torna-se ainda mais importante, e exige um preparo e uma experiência ainda maiores (CARDIM et al., 2009; CORREIA; LINHARES, 2008). O método de avaliação da dor deve ser condizente com a faixa etária da criança. Portanto, nesses casos, a escala NIPS para crianças menores de dois anos de idade, já preconizada na instituição, deveria ser utilizada, pois possibilita uma avaliação mais eficaz e fidedigna através de parâmetros fisiológicos e comportamentais (LAWRENCE et al., 1993), entretanto, apenas alguns profissionais citaram a escala de NIPS durante a entrevista.

Em três estudos realizados em um berçário de cuidados intensivos de uma maternidade pública, em uma unidade de cuidados intensivos neonatais de um hospital universitário, e em uma unidade pediátrica também de um hospital universitário, foi relatado pelos profissionais de enfermagem que na impossibilidade da criança relatar sua dor, o choro é o comportamento mais utilizado na avaliação, além de fácies de dor, gemência, irritabilidade e resposta motora. Alguns profissionais ainda citam as alterações fisiológicas, como frequência cardíaca e respiratória (VERONEZ; CORRÊA, 2010; PAIXÃO et al., 2011; SILVA et al., 2011). 
Entretanto, como citado anteriormente, a observação desses sinais comportamentais e fisiológicos deve ser padronizada através de uma escala de avaliação da dor, o que não é observado no presente estudo, no qual um terço dos participantes referiram não utilizar nenhuma escala, e avaliar a dor na criança apenas por meio da observação de suas reações como o choro, a face, as alterações de comportamento e de sinais vitais, o que torna a avaliação da dor muito subjetiva, podendo variar de acordo com o profissional que a está realizando. Alguns poucos profissionais afirmaram ainda utilizar o método da "eliminação", descartando todas as possíveis causas de desconforto da criança (fome, frio, troca de fralda etc.), para depois considerar a dor. A falta de utilização das escalas para avaliação da dor, também foi observada em outros estudos, nos quais muitos profissionais da equipe de enfermagem reconhecem a existência dessas escalas, mas confessam que muitas vezes não as utilizam (PAIXÃO et al., 2011; SILVA et al., 2011).

Apesar disso, avaliação da dor foi considerada por toda a equipe de enfermagem como sendo algo de grande importância. A maioria refere que essa avaliação traria um maior conforto e bem estar ao paciente, uma vez que poderia ser realizada uma analgesia mais eficaz.

Ah, eu acho que é de fundamental importância, para você estar fazendo uma analgesia numa criança. Porque não é só a bactéria, o antibiótico tem que ter um analgésico, um suporte. Serve como um suporte para estar auxiliando na recuperação. (TEC. 1)

Avaliar a dor como $5^{\circ}$ sinal vital é uma forma de melhorar a qualidade de vida do cliente, pois esta rotina viabiliza o planejamento dos horários de medicação de acordo com a necessidade de cada paciente, evitando assim que o mesmo sinta dor, e contribuindo para seu bem estar (PEDROSO; CELICH, 2006). A dor, quando não tratada, pode trazer prejuízos físicos e psicológicos para a criança.
Afetando seu desenvolvimento e a maneira como ela enfrentará futuras experiências dolorosas, uma vez que a criança também possui memória para a dor (FINLEY et al., 2005; VERONEZ; CORRÊA, 2010).

\section{Fragilidades no Manejo da Dor da Criança}

Porém, alguns profissionais de enfermagem ainda acreditam na hipótese de que a criança pode estar mentindo sobre sua dor, e que a avaliação serve para verificar se a dor é verdadeira ou não.

A gente que trabalha a noite percebe muito isso. Tomou, gostou de tomar o remédio para dormir, daí no outro dia está com uma dor naquele horário. (AOP. 1)

É importante porque é uma maneira da gente avaliar. A dor que a criança está... até que ponto a criança está com aquela dor. Até que ponto é verdade da criança a dor também. Porque também tem disso, tem muita criança que fala que está com dor. Está com dor mas está rindo, está... aí não está com dor. (AUX. 5)

De acordo com a definição da Associação Internacional para Estudos da Dor a dor é aquilo que a pessoa diz que é, e existe sempre que ela assim o disser (MERSKEY; BOGDUK, 2012, p. 211-212). Portanto, cabe a equipe de saúde acreditar nas palavras do paciente, independente da idade do mesmo. Muitos cuidadores afirmam que, às vezes, o paciente só refere sentir dor para chamar a atenção, ou porque está se sentindo ansioso e carente, porém mesmo nessas situações, cabe a equipe acreditar nele, rever suas necessidades e cuida-lo de maneira que essa dor seja amenizada, oferecendo conforto, carinho e atenção (PEDROSO; CELICH, 2006). O alívio da dor é um direito da criança e precisa ser respeitado (SILVA et al., 2011).

A própria equipe de enfermagem, ao fazer uma análise da avaliação da dor que vem sendo realizada no setor de foram geral, reconhece que ainda há muito que melhorar. A maioria dos 
entrevistados refere que muitos colegas não realizam a avaliação em todos os horários, não dando a importância devida à dor da criança. Este dado contradiz outros dados encontrados, como o fato de todos os participantes terem afirmado realizar a avaliação da dor como $5^{\circ}$ sinal vital e considerar essa avaliação de grande importância para o bem-estar da criança.

Tem gente que não sabe avaliar, tem gente que olha, a criança está chorando, está se estremecendo todo de dor e fala que a criança não está com dor. (AUX. 5)

Eu acho que é a consciência mesmo da equipe, a gente estar trabalhando numa conscientização, tirando mais as dúvidas... apesar de ter sido feito vários treinamentos, eu acho que é trabalho de formiguinha, tem que estar voltando sempre... na mesma... até que seja atingido realmente como $5^{\circ}$ sinal vital. (ENF. 2)

Um estudo realizado com enfermeiras de uma UTI Pediátrica mostrou que a sensibilização é de extrema importância para que o enfermeiro comprometa-se com o alívio da dor. O profissional percebe o paciente com dor e se sensibiliza com sua situação, todas as experiências dolorosas vividas por ele vêm à tona nesse momento, e então ele se preocupa em aliviar a dor de seu paciente (VIANNA; DUPAS; PEDREIRA, 2006).

A equipe de enfermagem está numa posição real para avaliar, intervir e evitar o aparecimento de novos episódios dolorosos na criança, a avaliação correta da dor subsidia a prática da enfermagem e possibilita um melhor tratamento e consequente alívio. Uma assistência efetiva à criança com dor baseia-se também no cuidado mais humanizado, na percepção de suas necessidades e no reconhecimento da individualidade de cada cliente (KANAI; FIDELIS, 2010).

Já em relação aos métodos não farmacológicos no tratamento da dor, a maioria disse não utilizar nenhum, controlando a dor apenas com a medicação prescrita. Dentre os profissionais que utilizam, os métodos mais citados foram as compressas, a glicose à $25 \%$ por via oral, o apoio emocional e o posicionamento.

Segundo outros estudos acerca do tema, esse são os métodos não farmacológicos mais conhecidos e utilizados pelos profissionais no controle da dor, porém, outros métodos eficazes como amamentação, presença dos pais em procedimentos dolorosos, massagem relaxante, toque terapêutico, uso de imagens, técnica de distração, uso de brinquedos, poderiam ser mais utilizados e divulgados, obtendo um resultado mais significativo (KANAI; FIDELIS, 2010; PAIXÃO, et al., 2011; QUEIROZ et al., 2007; SILVA et al., 2011; VERONEZ; CORRÊA, 2010).

Os métodos não farmacológicos não substituem o tratamento com medicação analgésica, mas considerando que a dor é muito mais do que uma sensação, e recebe influências psicológicas, sociais, emocionais, entre outras, a associação desses métodos no tratamento teria grande relevância no controle da dor, muitas vezes levando a um alívio mais rápido do sintoma (LEÃO et al., 2008; TACLA; HAYASHIDA; LIMA, 2008).

Apesar dos benefícios dessa associação de tratamentos, outros estudos também afirmam que o tratamento mais utilizado pela equipe continua sendo o farmacológico. Muitas vezes, diante da queixa de dor, o profissional de enfermagem apenas verifica se há analgésicos prescritos pela equipe médica, deixando de ter uma visão mais holística da situação e de realizar outras medidas para promover o alívio e conforto da criança (CARDIM et al., 2009; KANAI; FIDELIS, 2010).

Porém, o mais preocupante com relação ao tratamento da dor é que uma parcela da equipe, ainda que pequena, considera e utiliza o placebo como método não farmacológico, acreditando que seja uma forma não só de controlar a dor na criança, mas também de verificar a veracidade da dor. 
Então, uma vez eu fiz aquele tal daquele placebo lá. Que a criança fala que está com dor, está com dor, você medica, dá um placebo e a criança.... ah, estou bem. Já fiz muitas vezes isso. (AUX. 1)

A gente dá água doce, pelo mando da... da supervisora. Disfarça, põe groselha... já aconteceu sim. Mas isso bem pouco. (AUX. 6)

$\mathrm{O}$ efeito placebo, em alguns casos, mostra-se eficaz, porém, a real importância deste método, bem como as respostas obtidas do tratamento da dor com placebos ainda estão mal definidas. Acredita-se que o efeito placebo é mediado por reações cerebrais que podem levar a melhora do corpo, mas diante das dúvidas acerca do uso do placebo, considera-se um grande erro usá-lo para verificar se a dor do paciente é real, especialmente quando se trata de uma criança. O profissional que usa do placebo para estes fins está apenas iludindo o seu paciente e negligenciando a queixa do mesmo, causando muitas vezes mais sofrimento e perdendo a sua confiança (DOURADO et al., 2004).

Além da avaliação e manejo adequado da dor infantil, a equipe de enfermagem deve se conscientizar de que a prevenção é fundamental. Medidas como evitar a manipulação desnecessária da criança, coletas de exames planejadas a fim de evitar punções desnecessárias, o uso de cateteres centrais em tratamentos prolongados, manutenção de um ambiente tranquilo e organizado, e presença da família podem evitar ou reduzir a dor na criança, assim como ajudá-la no enfrentamento da situação dolorosa (PAIXÃO et al., 2011; VERONEZ, CORRÊA, 2010).

Na relação entre a equipe de enfermagem, criança e família, deve existir credibilidade e sensibilidade para que a assistência seja humanizada e de qualidade. Para que isso aconteça, é fundamental que a equipe conheça as escalas de avaliação da dor de acordo com a faixa etária e condição clínica do paciente, e as aplique conforme o preconizado, dando-lhes a devida importância no processo que resultará no tratamento e alívio da dor (KANAI; FIDELIS, 2010; SILVA et al., 2011).

\section{Considerações Finais}

Foi possível perceber que ainda falta conhecimento e conscientização sobre a dor para a equipe de enfermagem. Alguns conceitos parecem já terem sido aceitos e serem praticados pelos profissionais, mas ainda há muito que melhorar. É necessário investir em educação continuada para os profissionais de enfermagem visando esclarecer dúvidas e rever conceitos errôneos em relação à dor pediátrica. $\mathrm{O}$ enfermeiro, como líder da equipe, deve continuamente supervisionar a correta utilização das escalas de dor e outros métodos de avaliação, bem como as estratégias empregadas para seu alívio, em especial as não farmacológicas, visto que independem de prescrição médica.

A criança, mais do que o adulto, depende de seu cuidador, e, portanto, cabe a ele identificar suas reações e intervir de forma correta aliviando o sofrimento e proporcionando um cuidado mais humanizado e de qualidade. Sem uma boa avaliação não há uma boa intervenção; e sem um controle adequado da dor, haverá tempos de internação mais prolongados e processos de hospitalização mais traumatizantes.

\section{Referências}

CARDIM, M. G.; SILVA, L. R.; NASCIMENTO, M. A. L.; BIESBROECK, F. C. C. Processo saúde-doença: um olhar para a dor da criança na perspectiva da enfermagem. Revista de Pesquisa: Cuidado é Fundamental, Rio de Janeiro, v. 1, n. 1, p. 65-70, 2009. Disponível em: <http://seer. unirio.br/index.php/ cuidadofundamental/article/ viewFile/283/276>. Acesso em: 25 abr. 2014.

CLARO, M. T. Escala de faces para avaliação da dor em crianças etapa preliminar. 1993. 60 f. Dissertação (Mestrado em Enfermagem) Universidade de São Paulo: Escola de Enfermagem de Ribeirão Preto, Ribeirão Preto, 1993.

CORREIA, L. L.; LINHARES, M. B. M. Avaliação do comportamento de crianças em situações de dor: revisão da literatura. Jornal de Pediatria, Rio de Janeiro, v. 84, n. 6, p. 477-86, 2008. 
DOURADO, E.; FERNANDES, T. C. A.; MEIRELES, J. S. S.; MONTEIRO, I. S. Dor e efeito placebo. Revista de Cirurgia e Traumatologia BuçoMaxilo-Facial, Recife, v. 4, n. 3, p. 197-203, 2004.

FINLEY, G. A. Supporting Evidence for Childkind Principles. 2010. Disponível em: $<$ http://aneswebout. tch.harvard.edu/sites/childkindinternational/ files/2013/05/EvidenceChildKindPrinciplesPort. pdf>. Acesso em: 25 abr. 2014.

FINLEY, G. A.; FRANCK, L. S.; GRUNAU, R. E.; VON BAEYER, C. L. Why children's pain matters. International Association for the Study of Pain. Pain: Clinical Updates, Washington, v. 13, n. 4, p. 1-6, 2005. Disponível em: <http://iasp.files.cms-plus.com/ Content/ContentFolders/Publications2/PainClinical Updates/Archives/PCU05-4_1390264071339_24. pdf $>$. Acesso em: 22 ago. $201 \overline{4}$.

HOCKENBERRY, M. J. Wong fundamentos de enfermagem pediátrica. 8. ed. Rio de Janeiro: Mosby, 2011. p. 162-202.

KANAI, K. Y.; FIDELIS, W. M. Z. Conhecimento e percepção da equipe de enfermagem em relação à dor na criança internada. Revista Dor, São Paulo, v. 11, n. 1, p. 20-7, 2010. Disponível em: <http://files.bvs. br/ upload/S/1806-0013/2010/v11n1/a1495.pdf $>$. Acesso em: 25 abr. 2014.

KAZANOWSK, M. K.; LACCETTI, M. S. Dor: fundamentos, abordagem, tratamento. Rio de Janeiro: Guanabara Koogan, 2005.

LAWRENCE, J.; ALCOCK, D.; MCGRATH, P.; KAY, J.; MACMURRAY, S. B.; DULBERG, C. The development of a tool to assess neonatal pain. Neonatal Network, United States, v. 12, n. 6, p. 5966, 1993.

LEÃO, E. R.; SILVA, C. P. R.; ALVARENGA, D. C.; MENDONÇA, S. H. F. (Org.). Qualidade em saúde e indicadores como ferramenta de gestão. São Caetano do Sul: Yendisa, 2008.

MAYAN, M. J. Una introducción a los métodos cualitativos: un módulo de entrenamiento para estudiantes y profesionales. México: Qual Institute Press, 2001.
MERSKEY, H.; BOGDUK, D. N. A current list with definitions and notes on usage. In:

Classification of chronic pain. Seattle: IASP Press, 2012. p. 207-214. Disponível em: <http://www.iasppain.org/Education/Content.aspx?ItemNumber $=169$ 8\&navItemNumber=576>. Acesso em: 22 ago. 2014.

NASCIMENTO, L. A.; KRELING, M. C. G. D. Avaliação da dor como quinto sinal vital: opinião de profissionais de enfermagem. Acta Paulista de Enfermagem, São Paulo, v. 24, n. 1, p. 50-4, 2011.

PAIXÃO, M. C. S.; MARANHÃO, T. A.; MELO, B. M. S.; VIEIRA, T. S.; MONTEIRO, C. F. S. A percepção da equipe de enfermagem sobre a dor do recém-nascido. Revista Interdisciplinar, Teresina, v. 4, n. 2, p. 16-20, 2011. Disponível em: <http:// uninovafapi.edu.br/sistemas/revistainterdisciplinar/ v4n2/pesquisa/ p2_v4n2.pdf $>$. Acesso em: 25 abr. 2014.

PEDROSO, R. A.; CELICH, K. L. S. Dor: Quinto sinal vital, um desafio para o cuidar em enfermagem. Revista Texto e Contexto-Enfermagem, Florianópolis, v. 15 , n. 2, p. 270-276, 2006.

PERSEGONA, K. R.; ZAGONEL, I. P. S. A relação intersubjetiva entre o enfermeiro e a criança com dor na fase pós-operatória no ato de cuidar. Escola Anna Nery, Rio de Janeiro, v. 12, n. 3, p. 430-6, 2008.

QUEIROZ, F. C.; NASCIMENTO, L. C.; LEITE, A. M.; SANTOS, M. F.; SCOCHI, C. G. S. Manejo da dor pós-operatória na enfermagem pediátrica: em busca de subsídios para aprimorar o cuidado. Revista Brasileira de Enfermagem, São Paulo, v. 60, n. 1, p. 87-91, 2007.

\section{SILVA ET AL., 2010 falta referencia}

SILVA, M. S.; PINTO, M. A.; GOMES, L. M. X.; BARBOSA, T. L. A. Dor na criança internada: a percepção da equipe de enfermagem. Revista Dor, São Paulo, v. 12, n. 4, p. 314-20, 2011. Disponível em: $<$ http://www.scielo.br/pdf/rdor/v12n4/a06v12n4. pdf $>$. Acesso em: 25 abr. 2014.

SILVA, Y. P.; GÓMEZ, R. A.; MÁXIMO, T. A.; SILVA, A. C. S. Avaliação da dor em neonatologia. Revista Brasileira de Anestesiologia, Rio de Janeiro, v. 57, n. 5, p. 565-74, 2007. 
TACLA, M. T. G. M.; HAYASHIDA, M.; LIMA, R. A. G. Registros sobre dor pós-operatória em crianças: uma análise retrospectiva de hospitais de Londrina, PR, Brasil. Revista Brasileira de Enfermagem, São Paulo, v. 61, n. 3, p. 289-95, 2008.

VERONEZ, M.; CORRÊA, D. A. M. A dor e o recém-nascido de risco: percepção dos profissionais de enfermagem. Cogitare Enfermagem, Curitiba, v. 15, n. 2, p. 263-70, 2010. Disponível em: $<$ http:// ojs.c3sl.ufpr.br/ojs/index.php/cogitare/article/ view/17859/11652>. Acesso em: 25 abril 2014.

VIANNA, D. L.; DUPAS, G.; PEDREIRA, M. L. G. A avaliação da dor da criança pelas enfermeiras da unidade de terapia intesiva. Pediatria, São Paulo, v. 28, n. 4, p. 251-61, 2006.

WALCO; GOLDSCHNEIDER, 2008 falta referência

WHALEY, L. F; WONG, D. L. Enfermagem pediátrica: elementos essenciais à intervenção efetiva. 5. ed. Rio de Janeiro: Guanabara Koogan, 2007. 